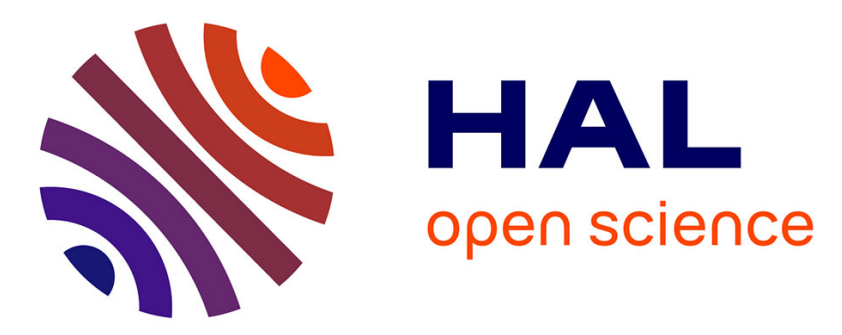

\title{
Helmholtz Tomography of ambient noise surface wave data to estimate Scholte wave phase velocity at Valhall Life of the Field
}

Aurélien Mordret, Nikolai M. Shapiro, Satish C. C. Singh, Philippe Roux, O.I. Barkved

\section{To cite this version:}

Aurélien Mordret, Nikolai M. Shapiro, Satish C. C. Singh, Philippe Roux, O.I. Barkved. Helmholtz Tomography of ambient noise surface wave data to estimate Scholte wave phase velocity at Valhall Life of the Field. Geophysics, 2013, 78 (2), pp.WA99-WA109. 10.1190/GEO2012-0303.1 · hal-00874171

\section{HAL Id: hal-00874171 \\ https://hal.science/hal-00874171}

Submitted on 22 Oct 2013

HAL is a multi-disciplinary open access archive for the deposit and dissemination of scientific research documents, whether they are published or not. The documents may come from teaching and research institutions in France or abroad, or from public or private research centers.
L'archive ouverte pluridisciplinaire $\mathbf{H A L}$, est destinée au dépôt et à la diffusion de documents scientifiques de niveau recherche, publiés ou non, émanant des établissements d'enseignement et de recherche français ou étrangers, des laboratoires publics ou privés. 


\title{
Helmholtz Tomography of ambient noise surface wave data to estimate Scholte wave phase velocity at Valhall Life of the
}

\section{Field}

\author{
Aurélien Mordret ${ }^{1}$, Nikolaï M. Shapiro ${ }^{1}$, Satish S. Singh ${ }^{2}$, Philippe Roux $^{3}$, and \\ Olav I. Barkved ${ }^{4}$ \\ ${ }^{1}$ Institut de Physique du Globe de Paris, Equipe Sismologie, CNRS UMR 7154, \\ Université Paris Diderot, Paris 7, Paris, France. (mordret@ipgp.fr, nshapiro@ipgp.fr) \\ ${ }^{2}$ Institut de Physique du Globe de Paris, Equipe Géoscience Marine (singh@ipgp.fr) \\ ${ }^{3}$ ISTerre, Grenoble, France (philippe.roux@obs.ujf-grenoble.fr) \\ ${ }^{4}$ BP Norge, Stavenger, Norway (olav-inge.barkved@no.bp.com)
}

(April 3, 2013)

Running head: Noise tomography at Valhall

\begin{abstract}
We applied the 'Helmholtz tomography' technique to 6.5 hours of continuous seismic noise record dataset of the Valhall Life of Field network. This network, that has 2320 receivers, allows us to perform a multi-frequency high-resolution ambient-noise Scholte wave phase velocity tomography at Valhall. First, we computed cross-correlations between all possible pairs of receivers to convert every stations into a virtual source recorded by all other receivers. Our next step was to measure phase travel-times and spectral amplitudes at different periods from cross-correlations between stations separated by distances between 2 and 6 wavelengths. This is done in a straightforward fashion in the Fourier domain.
\end{abstract}


Then, we interpolated these measurements onto a regular grid and computed local gradients of travel times and local Laplacians of the amplitude to infer local phase velocities using a frequency dependent Eikonal equation. This procedure was repeated for all 2320 virtual sources and final phase velocities were estimated as statistical average from all these measurements at each grid points. The resulting phase velocities for periods between 0.65 and $1.6 \mathrm{~s}$ demonstrate a significant dispersion with an increase of the phase velocities at longer periods. Their lateral distribution is found in very good agreement with previous ambient noise tomography done at Valhall as well as with a full waveform inversion $\mathrm{P}$-wave model computed from an active seismic dataset. We put efforts on assessing the spatial resolution of our tomography with checkerboard tests and we discussed the influence of the interpolation methods on the quality of our final models. 


\section{INTRODUCTION}

The development of very dense seismic arrays consisting of several hundreds or thousands of sensors have lead to the emergence of a new method of surface wave tomography. This method, first described by Lin et al. (2009) as 'Eikonal tomography', was later generalized by Lin and Ritzwoller (2011) as a 'Helmholtz tomography'. It is based on tracking of surface wave fronts across a seismic array, dense enough to properly sample the wave field, and on measuring the local gradients of wave travel times and amplitudes which result in direct computation of local phase speeds through the application of a frequency dependent Eikonal equation. This method is more accurate than standard straight-ray tomography because it accounts for bent rays and finite frequency effects (Lin et al., 2009). Helmholtz tomography can be applied to data from earthquakes (e.g. Lin and Ritzwoller, 2011) or active seismic sources (e.g. Gouédard et al., 2012) as well as inter-station correlations of the ambient seismic noise (e.g. Gouédard et al., 2008, and references therein) data.

It has been shown both empirically (Campillo and Paul, 2003; Shapiro and Campillo, 2004) and theoretically (e.g., Weaver and Lobkis, 2001; Wapenaar, 2004; Gouédard et al., 2008) that the cross-correlation of a random wave field, like seismic noise, recorded at two sensors provides a Green function between these two sensors. Therefore, computing noise cross-correlation results in large quantity of surface wave data when each sensor of an array can be seen as a virtual seismic source recorded by all other receivers. For receivers at the earth's surface, these cross-correlations are dominated by the fundamental-mode surface waves (e.g. Shapiro and Campillo, 2004; Kimman and Trampert, 2010). Many researchers have used information extracted from noise cross-correlations to perform ambient noise surface wave tomographies in different regions around the world (e.g. Shapiro et al., 2005; 
Sabra et al., 2005; Moschetti et al., 2007; Lin et al., 2007; Yang et al., 2007; Lin et al., 2008; Zheng et al., 2008; Stehly et al., 2009). On a local scale, Brenguier et al. (2007) succeded in imaging a volcanic edifice on the La Réunion Island. This technique has also been applied to exploration geophysics problems for imaging the offshore shallow subsurface (Bussat and Kugler, 2011; de Ridder and Dellinger, 2011; Mordret et al., 2012) or to retrieve reflection response of onshore sedimentary basins (Draganov et al., 2007, 2009).

For this study, we used a dataset from the Valhall Life of Field Seismic (LoFS) network situated in the North Sea. The LoFS network was the world largest permanent ocean-bottom-cable array at the time of its installation in 2003. It consists of 2320 fourcomponent sensors (east, north and vertical geophpones plus an hydrophone) installed on the seafloor (70 m water depth) over the Valhall oil reservoir (e.g. van Gestel et al., 2008, Fig. 1). An Eikonal tomography has already been applied to the Valhall data by de Ridder and Dellinger (2011) to compute a group velocity map of Scholte waves estimated from the cross-correlation of noise data without taking into account the wave dispersion. Mordret et al. (2012) performed a standard ambient noise surface wave tomography using the vertical-to-vertical component cross-correlations and computed group velocity maps at different periods. They obtained results that are comparable with those of de Ridder and Dellinger (2011). Muyzert et al. (2002) already used Scholte waves from active sources to estimate a 1D shear-wave velocity model of the shallow subsurface before the LoFS network installation. In the present paper, we apply the Helmholtz tomography to phase travel time of Scholte waves and obtain a multi-frequency high-resolution Scholte-wave phase velocity model, at periods between $0.65 \mathrm{~s}$ and $1.6 \mathrm{~s}$, from noise data recorded at the Valhall LoFS network. We particularly focus on two aspects of the Helmholtz tomography. First, we systematically assess the influence of the applied interpolation method by exploring the 
role of the tension coefficient which controls the smoothness of the interpolated surfaces. Second, we perform checkerboard tests to evaluate the spatial resolution of the Helmholtz tomography with an approach complementary to Lin et al. (2009).

\section{DATA AND NOISE CROSS-CORRELATIONS}

The 2320 4C-sensors of the Valhall LoFS network record 250 samples per second with a low-cut filter which removes most of the energy at periods longer than $2.5 \mathrm{~s}$. Inter-sensor spacing along cables is $50 \mathrm{~m}$ and inter-cable distance is $300 \mathrm{~m}$ (Fig. 1). We used $400 \mathrm{~min}$ ( $\sim 6.5$ hours $)$ of continuous records from these $23204 \mathrm{C}$ sensors.

We computed cross-correlations between all pairs of sensors (2 690040 total). The noise series processing and computation of cross-correlations is described in detail by (Mordret et al., 2012) and partly follows the workflow of Bensen et al. (2007). Following Mordret et al. (2012), who found that the seismic noise sources on vertical components were roughly homogeneously distributed with respect to the azimuth between $0.5 \mathrm{~s}$ and $2.85 \mathrm{~s}$ period, we whitened the records in this band before the correlation. However, we did not apply a temporal normalization because there was no strong temporal amplitude variations in the records. Therefore, our cross-correlations preserved the absolute amplitude information at all stations and for every component. In this study we only used Scholte waves on the vertical-vertical (ZZ) component because they present a much higher signal-to-noise ratio $(\mathrm{S} / \mathrm{N})$. Figure 2 shows the ZZ cross-correlations with respect to station 595 filtered between 0.6 and $2.85 \mathrm{~s}$. We observe a clear signal propagating from the station 595 with an average group velocity around $375 \mathrm{~m} / \mathrm{s}$. 


\section{HELMHOLTZ TOMOGRAPHY: METHOD AND IMPLEMENTATION}

The main idea of the method is that, in a smoothly heterogeneous medium, the propagation of a single surface wave mode at a single frequency can be approximated with a twodimensional Helmholtz equation (e.g. Friederich et al., 2000). By separating amplitude and phase of the wave in the Helmholtz equation, a frequency-dependent Eikonal equation can be derived (Biondi, 1992):

$$
\frac{1}{c_{i}(\omega, \mathbf{r})^{2}}=\left|\nabla \tau_{i}(\omega, \mathbf{r})\right|^{2}-\frac{\Delta A_{i}(\omega, \mathbf{r})}{A_{i}(\omega, \mathbf{r}) \omega^{2}}
$$

where $\tau$ and $A$ are the travel time and the spectral amplitude of the wave, respectively, and $c$ is the phase velocity, $\omega$ is the frequency, $\mathbf{r}$ is the position and $i$ denotes the virtual source. In this study we have 2320 virtual sources. With this equation, we can compute the phase velocities at all points from the local gradient $\left(\nabla=\frac{\partial}{\partial x}+\frac{\partial}{\partial y}\right)$ of the travel time and the Laplacian $\left(\Delta=\nabla^{2}=\frac{\partial^{2}}{\partial x^{2}}+\frac{\partial^{2}}{\partial y^{2}}\right)$ of the amplitude. This operation can be repeated for every virtual source $i$ and a final phase velocity map is computed as an average from these realizations.

The data processing is performed at distinct frequencies closely following the approach of Lin and Ritzwoller (2011), and is divided in four steps: first, a station $i$ is treated as a virtual source and cross-correlations between this station and all others are interpreted as virtual seismograms (shot gather). At this stage, a waveform selection based on a set of quality criteria such as $\mathrm{S} / \mathrm{N}$ is applied. Second, the selected correlations are used to measure the phase travel-times $\tau_{i}(\omega, \mathbf{r})$ between station $i$ and all other selected stations. For this step, we do not follow Lin et al. (2009), we preferred a more straightforward approach in the Fourier domain. The spectral amplitude $A_{i}(\omega)$ of the correlations is also measured at this stage. Third, the travel-times and amplitudes are interpolated onto a regular grid to 
allow the computation of the travel-time gradient and of the amplitude Laplacian. Fourth, the set of gradient maps, corrected from the set of Laplacian terms, are averaged to give the final phase velocity map and its uncertainty. The resolution of the method is accessed with checkerboard tests.

\section{Waveform selection}

Before measuring the amplitudes and the travel-times at a certain frequency, we reject lowquality waveforms based on the following criteria: first, symmetric correlations (average of the positive and negative sides) filtered between 0.67 and $2.85 \mathrm{~s}$ with a $\mathrm{S} / \mathrm{N}$ lower than 1.5 are discarded (e.g. white strips in Figure $3 \mathrm{~A}$ ). We estimate the $\mathrm{S} / \mathrm{N}$ as the ratio between the maximum absolute value in the move-out window demarcating the signal of interest (black lines in Figure 3A) and three times the standard deviation of the signal outside the window. The move-out window is defined by $t=D / v_{1}-1.1 \mathrm{~s}$ with $v_{1}=500 \mathrm{~m} / \mathrm{s}$ for the upper bound and $t=D / v_{2}+2.5 \mathrm{~s}$ with $v_{2}=330 \mathrm{~m} / \mathrm{s}$ for the lower bound, $D$ being the inter-station distance. We selected this low $\mathrm{S} / \mathrm{N}$ threshold to favour the homogeneous spatial data coverage. Second, we reject correlations for inter-station distances smaller than 2 wavelengths to avoid near source effects and for inter-station distances larger than 6 wavelengths ( purple dashed lines in Figure 4 for $0.8 \mathrm{~s}$ ) because it appears that the quality of the travel-time measurements drops significantly beyond this distance (Figure 4). We take a phase speed of $400 \mathrm{~m} / \mathrm{s}$ as a rule of thumb to calculate the wavelength. Third, we measure group travel-times as the time of the maximum of the envelope of the correlation filtered between 0.67 and $2.85 \mathrm{~s}$ and discard all correlations where the group velocity on the positive and negative sides of the correlation differs by more than $50 \mathrm{~m} / \mathrm{s}$. The symmetric part of the remaining correlations is used for the phase travel time measurement. Finally, 
we do not consider stations where there are less than 30 travel-time measurements after the selection. The above criteria result in rejecting $20-50 \%$ of the correlations depending on the period.

\section{Measurement of spectral amplitudes and phase travel-times}

We use symmetrical parts of the selected cross-correlations to measure phase travel times. We take Fourier transforms of the signals between black lines in Figure 3A to extract their spectral amplitudes and phases (Figure 3B). For an angular frequency $\omega$, the spectral phases of a single-mode surface wave $\varphi$ can be written (Lin et al., 2008) as:

$$
\varphi(\omega)=-\omega t_{c}(\omega)+2 n \pi+\varphi_{0},
$$

where $2 n \pi$ is the intrinsic $2 \pi$ phase ambiguity and $\varphi_{0}$ is the initial phase term (e.g. Lin et al., 2008). As shown in Figure 3 B-3C, the phase is unwrapped prior to the computation of the frequency dependent phase travel-time $t_{c}(\omega)$ :

$$
t_{c}(\omega)=\frac{-\varphi(\omega)+2 n \pi+\varphi_{0}}{\omega} .
$$

Since our final goal is to estimate the gradient of the phase travel-time, we do not need to find the exact initial phase which we set to an arbitrary constant value (the y-intercept of the best fitting line of the phase travel-time measurements). The resulting travel-times for station 595 are shown in Figure 3C and are superimposed on cross-correlation waveforms (Figure 4). For the period $0.8 \mathrm{~s}$ (purple dots) we show the full travel-time measurements for all distances. A clear trend at inter-station distance between 2 and 6 wavelengths 
(shown with the vertical dashed lines) degrades at larger inter-station distances. A similar behaviour is observed at all periods. We note that the average phase velocity (slope of the travel time with distance) increases with the period.

\section{Interpolation of spectral amplitudes and phase travel-times}

To compute the spatial gradient of the travel time and the spatial Laplacian of the amplitude, we need to interpolate the measurements obtained at station locations (Figure 5A and E) onto a regular spatial grid. The travel-time measurements and the amplitude measurements do not show the same robustness. Therefore we used two different interpolation schemes. Since the travel-time measurements are rather robust, we used an interpolation method that creates a surface passing through all the data points. On the other hand, the amplitude measurements are more scattered and we need an interpolation method which creates a surface passing between the data points in a smooth manner.

For the travel times, we used a spline-in-tension interpolation scheme (Wessel and Bercovici, 1998) on a $50 \mathrm{~m} \times 50 \mathrm{~m}$ regular grid across the whole Valhall array (Figure 5B-C). This interpolation mimics an elastic membrane with flexural rigidity passing through every measurement points with a tension applied at the boundaries. It is possible to introduce a non-dimensional fitting parameter that represents the portion of the strain energy resulting from tension relative to the total strain energy of the membrane. We call hereafter this parameter as the 'tension coefficient', it ranges between 0 and 1 (Wessel and Bercovici, 1998). By changing this tension coefficient, it is possible to vary the smoothness of the interpolated surface between the data points.

We follow Lin et al. (2009) to delete the zones that are not constrained by data by 
removing the areas where the difference between two different interpolations with two tension coefficients differing by $10 \%$ (0.07 and 0.063 in this study) deviate by more than 0.004 s. To avoid spurious oscillations of the travel-time surface along the cable direction, we remove the areas where the absolute curvature of the surface (taken as the Laplacian of the travel-time surface) is larger than $0.004 \mathrm{~s}^{2} / \mathrm{m}^{2}$. As an additional quality control, we remove measurements from location that are not surrounded by at least four locations with measurements. The remaining measurements (Figure 5B) are then re-interpolated with a tension coefficient of 0.07 and we keep only the region which enclose all the discrete traveltime measurements. After obtaining the final phase travel-time surface for the station $i$ (Figure 5C) we compute the squared gradient to obtain the Eikonal phase slowness map for the $i^{\text {th }}$ source (Figure 5D).

To evaluate the second term on the right-hand side of Equation 1 involving the Laplacian computation, hereafter called the 'amplitude term', we used a 'thin-plate spline in tension' method (e.g. Bookstein, 1989; Boer et al., 2001). The Laplacian computation is very sensitive to any measurement error and therefore we use a surface-fitting method that smooths the data, passing between the data points in a least-square sense, to avoid spurious oscillations of the estimated Laplacian.

Let $y_{k}, k=1, \ldots, N$ be the $N$ discrete measurement values at locations $x_{k}$ (Figure $5 \mathrm{E}$ ), in the case of a bivariate thin-plate spline, the measurements are modelled as

$$
y_{k}=f\left(x_{k}\right)+\varepsilon\left(x_{k}\right),
$$

where $f$ is an unknown smooth function and $\varepsilon\left(x_{k}\right)$ are random errors. The function $f$ is determined by minimizing the quantity 


$$
\sum_{k=1}^{N}\left|y_{k}-f\left(x_{k}\right)\right|^{2}+\lambda I(f)
$$

with:

$$
I(f)=\iint_{-\infty}^{+\infty}\left[\left(\frac{\partial^{2} f}{\partial x^{2}}\right)^{2}+2\left(\frac{\partial^{2} f}{\partial x \partial y}\right)^{2}+\left(\frac{\partial^{2} f}{\partial y^{2}}\right)^{2}\right] \mathrm{d} x \mathrm{~d} y
$$

where $\lambda$ is a smoothing parameter which controls the closeness of the surface to the data, it may vary from 0 to 1 . We take $\lambda=0.5$. For $\lambda>0.5$ the Laplacian of the amplitude starts to diverge, for $\lambda<0.5$ the smoothing is too strong and the amplitude term becomes negligible. The regions where the Laplacian of the amplitude is larger than $A \omega^{2} / c_{0}^{2}$ with $c_{0}=400 \mathrm{~m} / \mathrm{s}$ are rejected. The amplitude terms are then computed (Figure $5 \mathrm{G}$ ) following Equation 1. The amplitude term map is removed from the Eikonal phase slowness map to obtain the final Helmholtz phase slowness map for the source $i$ (Figure 5F). We see that the correction to the slowness map is very small in general. This can be due to the small amplitude variations in space as well as to the smoothing which blurs the Laplacian amplitude peaks. When this processing is done for all stations, we end up with a set of 2320 overlapping maps of estimated slownesses.

\section{Computing the final phase velocity map}

Phase slowness maps obtained from individual virtual sources are very noisy because of strong random measurement errors and biases caused by the interpolation methods. Therefore, following Lin et al. (2009), we determine the mean of the slowness distribution $S(\mathbf{r})$ and the standard deviation of the mean slowness $\sigma_{S}(\mathbf{r})$ from the individual slowness maps $s_{i}(\mathbf{r})$ with 


$$
\begin{gathered}
S(\mathbf{r})=\frac{1}{N(\mathbf{r})} \sum_{i=1}^{N(\mathbf{r})} s_{i}(\mathbf{r}), \\
\sigma_{S}(\mathbf{r})^{2}=\frac{1}{N(\mathbf{r})(N(\mathbf{r})-1)} \sum_{i=1}^{N(\mathbf{r})}\left(s_{i}(\mathbf{r})-S(\mathbf{r})\right)^{2},
\end{gathered}
$$

where $N(\mathbf{r})$ is the number of measurements at every location point. After initial calculation we perform an outlier rejection in two steps. First, we remove individual velocity maps with their mean values $m_{c_{i}}=\sum_{\mathbf{r}} c_{i}(\mathbf{r})=\sum_{\mathbf{r}} 1 / s_{i}(\mathbf{r})$ deviating from the global mean velocity $M_{C}=\sum_{\mathbf{r}} 1 / S(\mathbf{r})$ by more than one standard deviation $\sigma_{C}=\sqrt{\frac{1}{j-1} \sum_{i=1}^{j}\left(m_{c_{i}}-M_{C}\right)^{2}}, j$ being the number of virtual sources. Second, we remove from every individual velocity maps all points when the deviation from the individual mean $m_{c_{i}}$ exceeds $\pm 2 \sigma_{c_{i}}$, where $\sigma_{c_{i}}=\sqrt{\frac{1}{k_{i}-1} \sum_{\mathbf{r}}\left(c_{i}(\mathbf{r})-m_{c_{i}}\right)^{2}}$ and $k_{i}$ is the number of locations with measurements for the virtual source $i$.

The isotropic phase velocity map $C(\mathbf{r})$ and its uncertainty, $\sigma_{C}(\mathbf{r})$, are then obtained as

$$
\begin{gathered}
C(\mathbf{r})=\frac{1}{S(\mathbf{r})}, \\
\sigma_{C}(\mathbf{r})=\frac{1}{C(\mathbf{r})^{2}} \sigma_{S}(\mathbf{r}) .
\end{gathered}
$$

The final phase velocity maps are estimated for the cells with the number of measurements $N(\mathbf{r})>40$ and $\sigma_{C}(\mathbf{r})<20 \mathrm{~m} / \mathrm{s}$.

\section{Spatial resolution}

The Helmholtz tomography method presented here does not involve inversion operation and the resolution cannot be evaluated with a standard matrix formalism (e.g. Tarantola, 2005). 
Lin et al. (2009) proposed a different approach to assess the length-scale of the features they could resolve at any points of their maps. They measured the statistical correlation of slowness measurements between pairs of location points. The correlation between point $i$ and every other points $k$ of the map varies from 1 (for correlation of point $i$ with itself) to 0 far from point $i$, exhibiting a cone-like shape centred at point $i$. Lin et al. (2009) took the base-radius of a best-fitting cone as the correlation length of the slowness measurement at point $i$ and showed that it was well correlated with the inter-station spacing.

In our study we decided to access the resolution of the final phase velocity maps with checkerboard tests. This approach, although non-optimal (Lévêque et al., 1993), gives an estimation of the global spatial resolution and of the anomaly distortion produced by a tomography. We only tested the resolution of the Eikonal tomography (Lin et al., 2009) by dropping the amplitude term in Equation 1 and by modelling the travel-times only. We constructed a checkerboard input model with successive smooth low and high velocity anomalies by using a 2D cosine function with $800 \mathrm{~m}$ wavelength and amplitudes varying from $380 \mathrm{~m} / \mathrm{s}$ to $420 \mathrm{~m} / \mathrm{s}$ to mimic a typical values obtained form the data in the Valhall region. We then used the multi-stencils fast marching method (MSFM, Hassouna and Farag, 2006) to solve the Eikonal equation and to compute travel times from the tested phase velocity distribution for all virtual sources in our dataset. We then used these synthetic travel times as input to the Eikonal tomography while keeping the same virtual sources and receivers as were selected for real data. The phase velocity maps computed via this Eikonal tomography are then compared with the input models. As discussed in following sections, the differences between the input and the estimated maps that characterize the systematic bias of the Helmholtz/Eikonal tomography are controlled by the station distribution and by the tension coefficient used during the interpolation of phase travel-times. Running the 
resolution tests over a range of this parameter allows us to select its optimal value.

\section{RESULTS}

Figs. 6A-D-G show Scholte wave phase velocity maps of the Valhall field obtained with the Helmholtz tomography at 3 different periods: $0.7 \mathrm{~s}, 1 \mathrm{~s}$ and $1.3 \mathrm{~s}$. The frames B-E-H and C-F-I show the uncertainty $\left(\sigma_{C}(\mathbf{r})\right)$ maps and number of measurements maps $(N(\mathbf{r}))$ maps, respectively. Our results are consistent with model obtained using different methods e.g. Sirgue et al. (2010); de Ridder and Dellinger (2011); Mordret et al. (2012). For a period of $0.7 \mathrm{~s}$, which is sensitive to very shallow structures $(100-150 \mathrm{~m})$, we observe high velocity anomalies corresponding to paleo-channels crossing the study area (dashed lines in Figure 6A), similar to Sirgue et al. (2010); de Ridder and Dellinger (2011); Mordret et al. (2012). The southern paleo-channel which cross the receiver network near its center is hardly visible because it goes through the central high velocity zone. This high velocity shallow zone is due to the contractional strain caused by the subsidence of the sea-floor induced by the reservoir compaction at depth (Hatchell et al., 2009). This high velocity area becomes less visible at longer period. At the south-east corner of the receiver network, a large meandering paleo-channel (dashed box in Figure 6D) is more clearly visible at $1 \mathrm{~s}$ period as it lays $\sim 100 \mathrm{~m}$ deeper than the small channels (Sirgue et al., 2010). On the 1 $\mathrm{s}$ map, we also start to see a very low velocity anomaly centred at $(\mathrm{x}, \mathrm{y})=(5.5 \mathrm{~km}, 7 \mathrm{~km})$ distance range which is even more anomalous at larger periods, e.g., on the $1.3 \mathrm{~s}$ map (dashed box in Figure 6G). This anomaly is present at all periods and is possibly related to a gas infiltration from the large gas cloud present above the reservoir. This anomaly is also observed on the FWI model (Sirgue et al., 2010). On average, the phase velocity constantly increases with the period as shown in Figure 8. The average group velocity dispersion curve 
from Mordret et al. (2012) is displayed for reference on the same Figure 8.

Our final phase velocity maps at short and intermediate periods do not cover the vicinity of the platforms where the velocity uncertainty is very high $(>20 \mathrm{~m} / \mathrm{s})$. This is explained by a poor measurement coverage due to the strong platform noise (Mordret et al., 2012).The checkerboard tests (Figure 7) show the input pattern (Figure 7B-E-H) is well reproduced in most of locations. The larger residuals appear in the vicinity of the platforms and in the areas where a line of station is missing. We discuss in more details this point in the next section. We performed tests with smaller-scale anomalies and we observed that the output images start to strongly degrade in the direction perpendicular to the cables when the lateral extent of the anomalies are smaller than 250-300 m, i.e., the inter-cable spacing.

\section{DISCUSSION}

The Helmholtz tomography is a powerful method for a very dense seismic networks. Its ability to obtain accurate phase velocity models in a straightforward fashion makes it very appealing for retrieving the shear velocity distribution in the shallow subsurface. When compared with the results of Mordret et al. (2012) obtained from a more "standard" straight-ray surface-wave tomography, the Helmholtz tomography shows similar geological features but the result is much less sensitive to the network imprint. The main difference observed between straight-ray tomography and Helmholtz tomography is that the later represents more accurately the wave propagation in a laterally heterogeneous medium. However, the Helmholtz tomography is not error-free. One of the most critical data processing steps used in our implementation of the Helmholtz tomography is the introduction of the interpolation of spectral amplitudes and phase travel-times, which introduces bias in the final phase velocity maps because it directly affects the estimation of the travel-time gradients and the 
Laplacian amplitude.

\section{Systematic errors due to the interpolation}

The results of the checkerboard tests (Figure 7) show a systematic disappearance of the low velocity anomalies at all period in the region where cables are missing (gray strips in Figure 1). This effect is particularly strong at the profiles on the edges of the receiver network but is also present to a lesser extent in the central part. This bias is not caused by a lack of stations because the measurement density maps do not show a systematic decrease of the number of measurements in the affected areas. Therefore the artefacts at the locations of missing lines are more likely due to the minimum curvature fitting scheme used for the travel-time interpolation. To constrain the interpolation in the areas where there is no data points, the interpolated map exhibits a minimum curvature behaviour and is thus very smooth. The gradient of this smooth map will then be small and the inverse of the gradient (the velocity) will be high. These artefacts may be modulated by the tension coefficient applied in the surface-fitting scheme.

We performed a simple test to assess the systematic biases created by the interpolation and the influence of the tension coefficient. We computed synthetic Eikonal tomographic images at different periods using a constant velocity model as input. Figure 9A shows the result for the $1 \mathrm{~s}$ period with a tension coefficient of 0.01 , which is virtually identical to the results at the other periods with the same tension coefficient. The resulting map shows strong high velocity artefacts along the missing sensor lines, plus thin low velocity artefacts on the edges of the network. The influence of the central platform is not really prominent and less systematic. The low velocity ring around the network is a side effect caused by the 
sharp truncation of the travel-time surface at the border of the resolved area.

A systematic exploration of different tension coefficient values shows that one can minimize the difference between the input constant velocity model and the output velocity map (Figure 9B). We use this optimal values of tension coefficients (0.07, red point in Figure 9B) to compute the final phase velocity maps. Figure $9 \mathrm{C}$ shows the output of the test with using the optimal tension coefficient. We see that the systematic errors have been reduced by a factor $>5$ compared to Figure 9A. The velocity artefacts induced by different values of the tension can easily reach $10 \mathrm{~m} / \mathrm{s}$ variations i.e. more than $15 \%$ of the velocity variations in the tomographic maps.

The role of the tension coefficient applied at the interpolation step is somehow similar to regularization applied during standard inversions. An advantage is that the optimal value of the tension coefficient is very straightforward to determine via the minimum of the RMS between the input and the output maps (Figure 9B), contrary to the strong ambiguity in selecting the regularization parameters in standard inversions.

\section{Helmholtz vs Eikonal tomography}

Figure 10 shows the difference between Helmholtz and Eikonal tomographic maps at $0.7 \mathrm{~s}$, $1 \mathrm{~s}$ and $1.3 \mathrm{~s}$. We see that in average the differences are of the order of $1 \mathrm{~m} / \mathrm{s}$ which is in some areas smaller than the bias introduced by the interpolation. The difference between the two methods tends to increase with the period as expected from Equation 1. When the frequency increases, the amplitude term in Equation 1 tends to become negligible. In our case, as a rule of thumb, we can consider the amplitude term negligible for periods below $1 \mathrm{~s}$. A particular feature is the ring of negative anomalies appearing around the central 
platform and to a lesser extent at long period around the south platform. These anomalies are limited to the vicinity of a small number of stations and a finer analysis would be needed to better understand their origin.

\section{CONCLUSION}

We applied the Helmholtz tomography technique to the particular case of the Valhall Life of Field network. To do so, we computed the Scholte-wave travel time between the stations in the Fourier domain, using the spectral phase information. We found that Helmholtz tomography, as a straightforward method that does not involve a formal inversion, is able to retrieve geological features that have been previously highlighted by full waveform inversion of an active seismic dataset. The spatial resolution assessed by checkerboard tests shows that one can recover features as small as $400 \mathrm{~m}$ wide but the resolution is limited in the direction perpendicular to the cable by the inter-cable distance. The receiver network geometry is the main source of artefacts which can be reduced by tuning the tension during the interpolation of measured travel times. In the case of the Valhall seismic network, if the tension coefficient is badly chosen, the systematic errors may overcome the corrections brought by the amplitude term of the frequency-dependent Eikonal equation. We propose to select the optimal value of this parameter via simple synthetic recovery tests. The Helmholtz tomography method may become a powerful technique to build a monitoring tool for the Valhall subsurface. We show that 6.5 hours of continuous are sufficient to produce multi-frequency high-resolution Scholte-wave phase velocity maps to image the near-surface geological objects. Repeating this procedure regularly in time would result in recovering the temporal variations in the isotropic and the anisotropic (Mordret et al., Azimuthal anisotropy at Valhall: the Helmholtz equation approach, in prep.) subsurface 
parameters. Our next step will be the depth inversion of the phase velocity maps at different periods to obtain a high resolution S-wave velocity model of the first hundred of meters of the Valhall subsurface. This model could be used as a starting model for a full waveform inversion process or to compute S-wave statics to correct for travel-times inconsistencies within seismic records.

\section{ACKNOWLEDGEMENTS}

We thank BP Norge AS and the Valhall partner, Hess Norge AS, for granting access to the seismic data. The authors also acknowledge G. Moguilny and S-CAPADE as well as the use of resources provided by the European Grid Infrastructure. For more information, please consult the EGI-InSPIRE paper (go.egi.eu/ pdnon). We would like to thank Joe Dellinger for preparing the data as well as for the discussions and comments he brought on an early version of this work. We thank Matthieu Landès for the fruitful discussions on tomographic resolution that helped to improve the quality of this work. We used the msfm2d.m Matlab code of D-J Kroon (www.mathworks.com/ matlabcentral/ fileexchange/ 24531-accuratefast-marching) to compute the checkerboard maps. We thank three anonymous reviewers for their constructive comments that help to improve the quality of this paper. The contribution of AM, NS, and PR was supported by a FP7 ERC Advanced grant 227507 (WHISPER). This is IPGP contribution number 3343. 


\section{REFERENCES}

Bensen, G., M. Ritzwoller, M. Barmin, A. Levshin, F. Lin, M. Moschetti, N. M. Shapiro, and Y. Yang, 2007, Processing seismic ambient noise data to obtain reliable broad-band surface wave dispersion measurements: Geophysical Journal International, 169, 12391260.

Biondi, B., 1992, Solving the frequency-dependent eikonal equation: Presented at the 1992 SEG Annual Meeting.

Boer, E., K. de Beurs, and A. Hartkamp, 2001, Kriging and thin plate splines for mapping climate variables: International Journal of Applied Earth Observation and Geoinformation, 3, 146-154.

Bookstein, F., 1989, Principal warps: Thin-plate splines and the decomposition of deformations: IEEE Transactions on Pattern Analysis and Machine Intelligence, 11, 567-585.

Brenguier, F., N. M. Shapiro, M. Campillo, A. Nercessian, and V. Ferrazzini, 2007, 3D surface wave tomography of the Piton de la Fournaise volcano using seismic noise correlations: Geophysical Research Letters, 34, 2305.

Bussat, S., and S. Kugler, 2011, Offshore ambient-noise surface-wave tomography above 0.1 hz and its applications: The Leading Edge, 30, 514 .

Campillo, M., and A. Paul, 2003, Long-range correlations in the diffuse seismic coda: Science, 299, 547 .

de Ridder, S., and J. Dellinger, 2011, Ambient seismic noise eikonal tomography for nearsurface imaging at Valhall: The Leading Edge, 30, 506.

Draganov, D., X. Campman, J. Thorbecke, A. Verdel, and K. Wapenaar, 2009, Reflection images from ambient seismic noise: Geophysics, 74, A63-A67.

Draganov, D., K. Wapenaar, W. Mulder, J. Singer, and A. Verdel, 2007, Retrieval of reflec- 
tions from seismic background-noise measurements: Geophysical Research Letters, 34, L04305.

Friederich, W., S. Hunzinger, and E. Wielandt, 2000, A note on the interpretation of seismic surface waves overthree-dimensional structures: Geophysical Journal International, $\mathbf{1 4 3}$ $335-339$.

Gouédard, P., L. Stehly, F. Brenguier, M. Campillo, Y. Colin de Verdière, E. Larose, L. Margerin, P. Roux, F. Sánchez-Sesma, N. M. Shapiro, and R. Weaver, 2008, Crosscorrelation of random fields: mathematical approach and applications: Geophysical prospecting, 56, 375-393.

Gouédard, P., H. Yao, F. Ernst, and R. van der Hilst, 2012, Surface wave eikonal tomography in heterogeneous media using exploration data: Geophysical Journal International, 1, 742.

Hassouna, M., and A. Farag, 2006, Accurate tracking of monotonically advancing fronts: Proceedings of the 2006 IEEE Computer Society Conference on Computer Vision and Pattern Recognition-Volume 1, IEEE Computer Society, 355-362.

Hatchell, P., P. Wills, and C. Didraga, 2009, Production Induced Effects on Near-surface Wave Velocities at Valhall: Presented at the 71st EAGE Conference and Exhibition.

Kimman, W., and J. Trampert, 2010, Approximations in seismic interferometry and their effects on surface waves: Geophysical Journal International, 182, 461-476.

Lévêque, J., L. Rivera, and G. Wittlinger, 1993, On the use of the checker-board test to assess the resolution of tomographic inversions: Geophysical Journal International, 115, $313-318$.

Lin, F., M. Moschetti, and M. Ritzwoller, 2008, Surface wave tomography of the western United States from ambient seismic noise: Rayleigh and Love wave phase velocity maps: Geophysical Journal International, 173, 281-298. 
Lin, F., and M. Ritzwoller, 2011, Helmholtz surface wave tomography for isotropic and azimuthally anisotropic structure: Geophysical Journal International, 186, 1104-1120.

Lin, F., M. Ritzwoller, and R. Snieder, 2009, Eikonal tomography: surface wave tomography by phase front tracking across a regional broad-band seismic array: Geophysical Journal International, 177, 1091-1110.

Lin, F., M. Ritzwoller, J. Townend, S. Bannister, and M. Savage, 2007, Ambient noise Rayleigh wave tomography of New Zealand: Geophysical Journal International, 170, 649-666.

Mordret, A., M. Landès, N. M. Shapiro, S. Singh, P. Roux, and O. I. Barkved, 2012, Studying seabed above the Valhall oil field with Ambient Noise Surface Wave Tomography: Geophysical Journal International, in press.

Moschetti, M., M. Ritzwoller, and N. M. Shapiro, 2007, Surface wave tomography of the western United States from ambient seismic noise: Rayleigh wave group velocity maps: Geochemistry Geophysics Geosystems, 8.

Muyzert, E., J. Kommedal, K. Iranpour, and B. Olofsson, 2002, Near surface s-velocities, statics, and anisotropy estimated from scholte waves: Presented at the EAGE 64th Annual Conference \& Exhibition.

Sabra, K., P. Gerstoft, P. Roux, W. Kuperman, and M. Fehler, 2005, Surface wave tomography from microseisms in Southern California: Geophysical Research Letter, 32.

Shapiro, N. M., and M. Campillo, 2004, Emergence of broadband Rayleigh waves from correlations of the ambient seismic noise: Geophysical Research Letter, 31, 1615-1619.

Shapiro, N. M., M. Campillo, L. Stehly, and M. Ritzwoller, 2005, High-resolution surfacewave tomography from ambient seismic noise: Science, 307, 1615.

Sirgue, L., O. Barkved, J. Dellinger, J. Etgen, U. Albertin, and J. Kommedal, 2010, Full 
waveform inversion: the next leap forward in imaging at Valhall: First Break, 28, 65-70.

Stehly, L., B. Fry, M. Campillo, N. M. Shapiro, J. Guilbert, L. Boschi, and D. Giardini, 2009, Tomography of the alpine region from observations of seismic ambient noise: Geophysical Journal International, 178, 338-350.

Tarantola, A., 2005, Inverse problem theory and methods for model parameter estimation: Society for Industrial Mathematics.

van Gestel, J.-P., J. H. Kommedal, O. I. Barkved, I. Mundal, R. Bakke, and K. D. Best, 2008, Continuous seismic surveillance of Valhall Field: The Leading Edge, 27, 1616-1621. Wapenaar, K., 2004, Retrieving the elastodynamic Green's function of an arbitrary inhomogeneous medium by cross correlation: Physical Review Letters, 93, 254301.

Weaver, R., and O. Lobkis, 2001, Ultrasonics without a source: Thermal fluctuation correlations at MHz frequencies: Physical Review Letters, 87, 134301.

Wessel, P., and D. Bercovici, 1998, Interpolation with splines in tension: a green's function approach: Mathematical Geology, 30, 77-93.

Yang, Y., M. Ritzwoller, A. Levshin, and N. M. Shapiro, 2007, Ambient noise Rayleigh wave tomography across Europe: Geophysical Journal International, 168, 259-274.

Zheng, S., X. Sun, X. Song, Y. Yang, and M. Ritzwoller, 2008, Surface wave tomography of China from ambient seismic noise correlation: Geochemistry Geophysics Geosystems, 9. 
Figure 1: Map of the Valhall network. Each blue dot is a 4C sensor. The large black circles show the location of the main platforms. The black star denotes the position of station 595 cited in the text. The gray strips denote missing lines of stations that are discussed in the last section. In the inset, the black cross shows the location of the Valhall field, with the bathymetry shown as the background.

Figure 2: Vertical-vertical (ZZ) component correlations between station 595 and all the other stations of the Valhall network filtered between 0.6 and $3 \mathrm{~s}$ and sorted with increasing inter-station distance.

Figure 3: Phase travel-time measurement processing at $0.8 \mathrm{~s}$ for station 595 . A) The symmetric correlations filtered between 0.67 and $2.85 \mathrm{~s}$ and windowed. The correlations with low SNR are rejected (white lines). B) Spectral phase of the correlations between 0.5 and $2 \mathrm{~Hz} . \mathrm{C})$ Travel-time as a function of the frequency and the inter-station distance computed with equation (3).

Figure 4: Travel-times measured at different periods for distances between 2 and 6 wavelengths for station 595 . For the $0.8 \mathrm{~s}$ period, we show the full distance measurements, the vertical purple dashed lines denote 2 and 6 wavelengths for $0.8 \mathrm{~s}$ period. The background image shows the windowed symmetric correlations from station 595 filtered between 0.67 and $2.85 \mathrm{~s}$. 
Figure 5: Helmholtz tomography procedure for station 595 at $0.8 \mathrm{~s}$. A) The discrete traveltimes. B) First interpolation of the travel-times: areas with to high curvature and unconstrained areas are removed (see text for details). C) Second interpolation of the travel-times. D) Magnitude of the spatial gradient of the travel-time surface representing the local slowness field. E) The discrete spectral amplitude measurements. F) The interpolated amplitude surface. G) The amplitude correction term computed from equation (1). H) The final local phase velocity map.

Figure 6: Final Helmholtz tomography maps: A), D), G): Phase velocity maps at 0.7, 1.0 and $1.3 \mathrm{~s}$ respectively. B), E), H): Velocity uncertainty maps at $0.7,1.0,1.3 \mathrm{~s}$ respectively. C), F), I): Number of measurement per cell at $0.7,1.0,1.3 \mathrm{~s}$ respectively. The dashed lines in frame A show the shallow paleo-channels, the dashed box in frame D shows the deeper large paleo-channel and the dashed box in frame $\mathrm{G}$ delineates the very low velocity anomaly mentioned in the text.

Figure 7: Checkerboard tests. B), E), H): Input velocity maps at 0.7, 1.0, $1.3 \mathrm{~s}$, respectively. A), D), G): Output velocity maps at $0.7,1.0$ and $1.3 \mathrm{~s}$, respectively. C), F), I): Residual maps at $0.7,1.0,1.3 \mathrm{~s}$, respectively. 
Figure 8: Average dispersion curves from phase velocities Helmholtz tomography (red curve) and standard ambient noise surface wave tomography group velocities (blue curve, after Mordret et al. (2012)).

Figure 9: A) Result of a synthetic test with a constant input velocity model of $400 \mathrm{~m} / \mathrm{s}$ and a tension coefficient of 0.01 at $1 \mathrm{~s}$ period. B) Misfit between the constant velocity model and the output tomography with respect to the tension applied in the surface-fitting interpolation scheme. The red dot shows the optimal tension coefficient C) Result for of a synthetic test with a constant input velocity model of $400 \mathrm{~m} / \mathrm{s}$ and the optimal tension coefficient of 0.07 at $1 \mathrm{~s}$ period.

Figure 10: The difference between Helmholtz tomography and Eikonal tomography at 0.7 $\mathrm{s}(\mathrm{A}), 1.0 \mathrm{~s}(\mathrm{~B})$ and $1.3 \mathrm{~s}(\mathrm{C})$. 


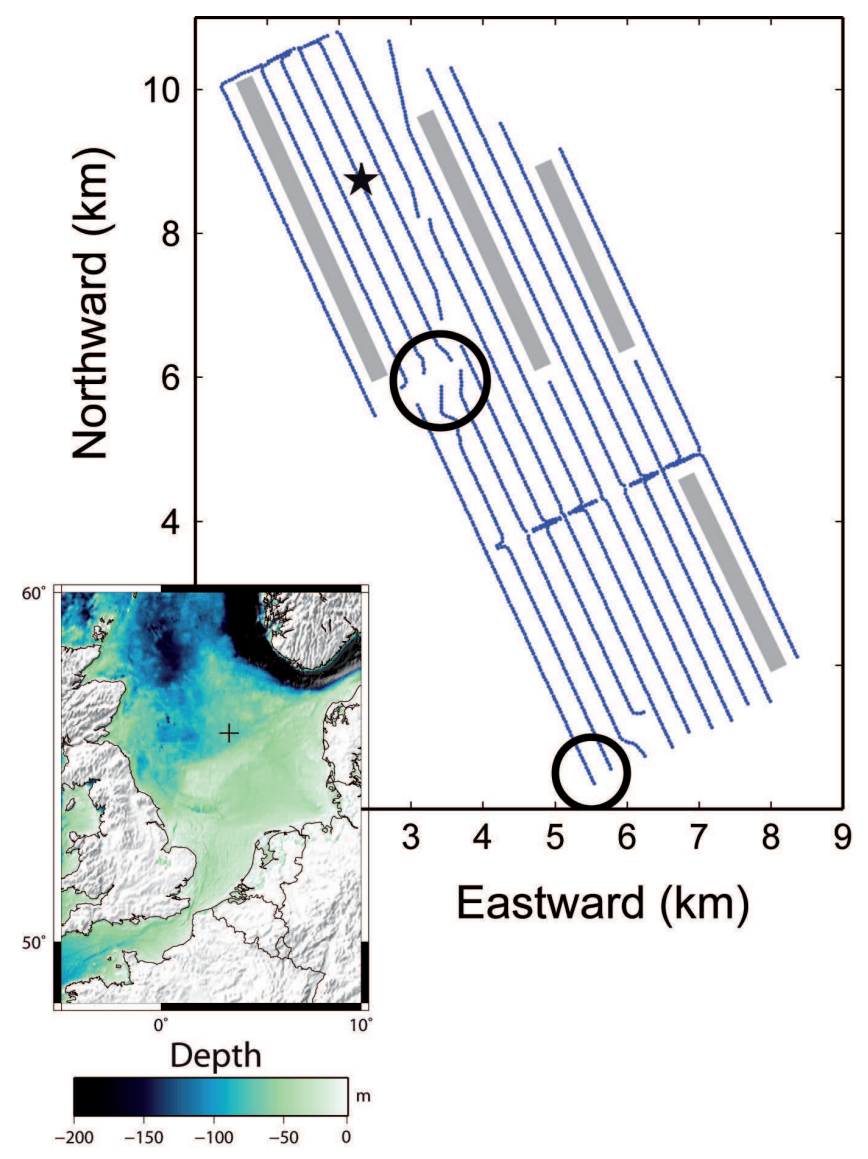

Figure 1

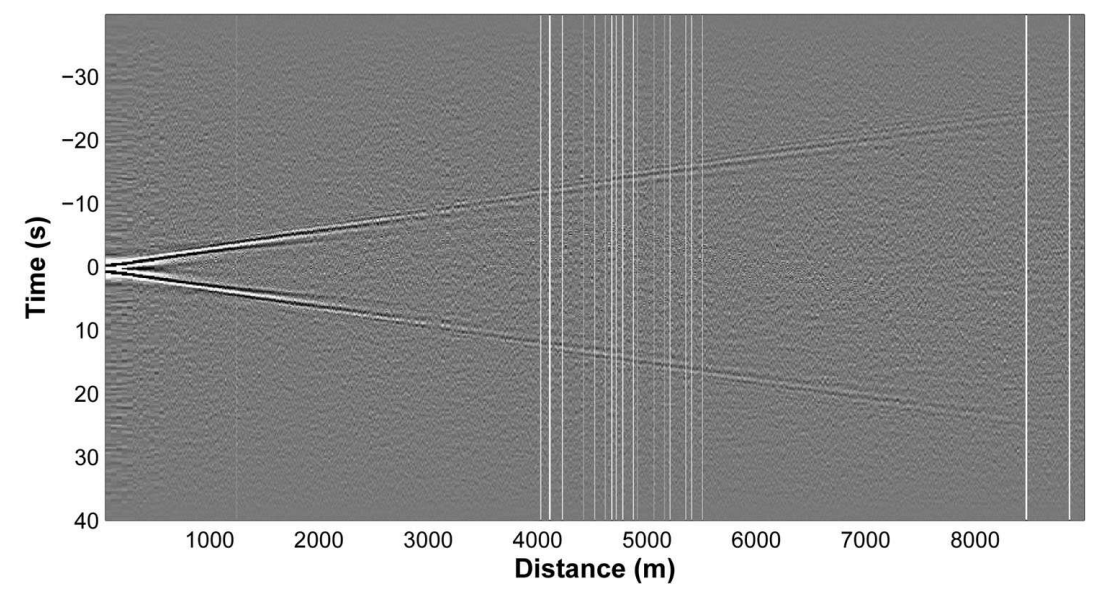

Figure 2 

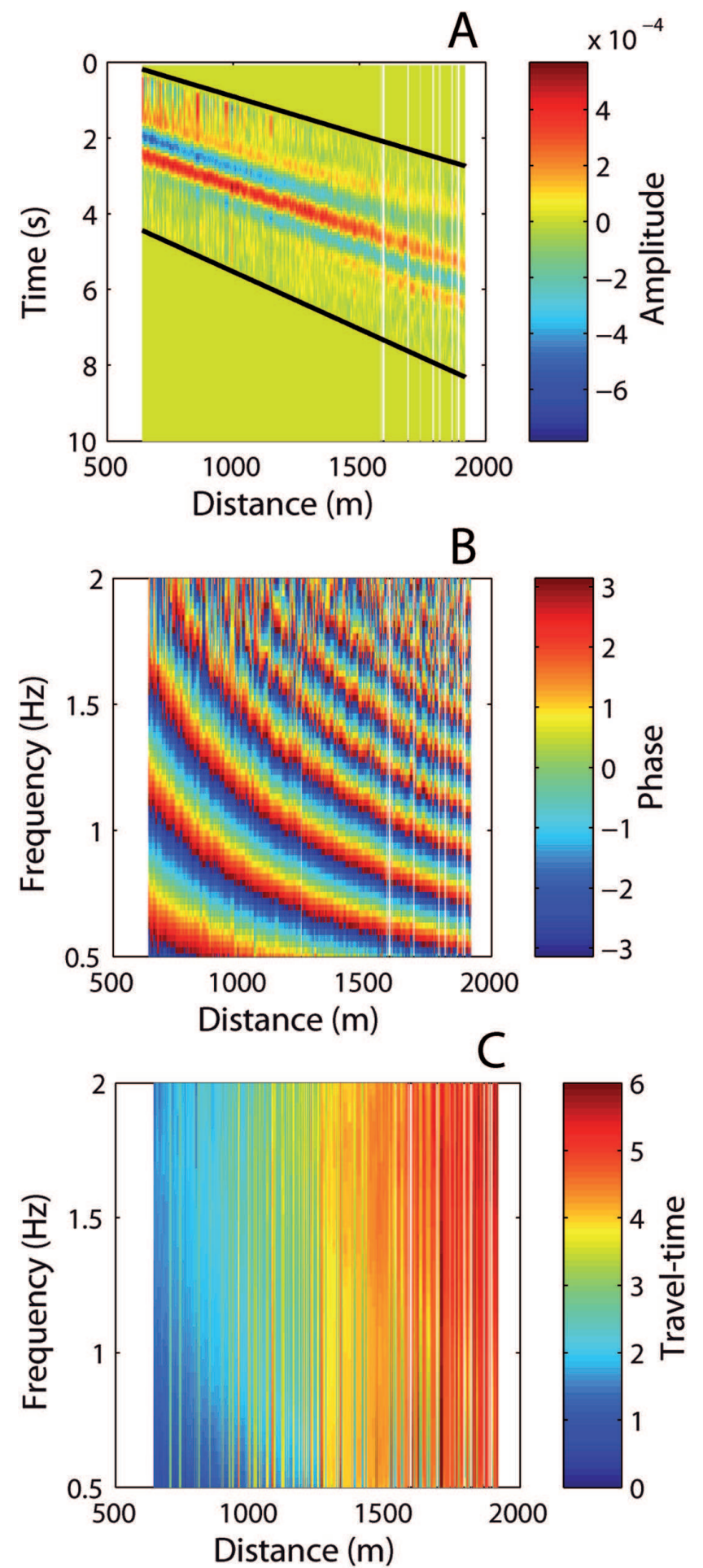

Figure 3 


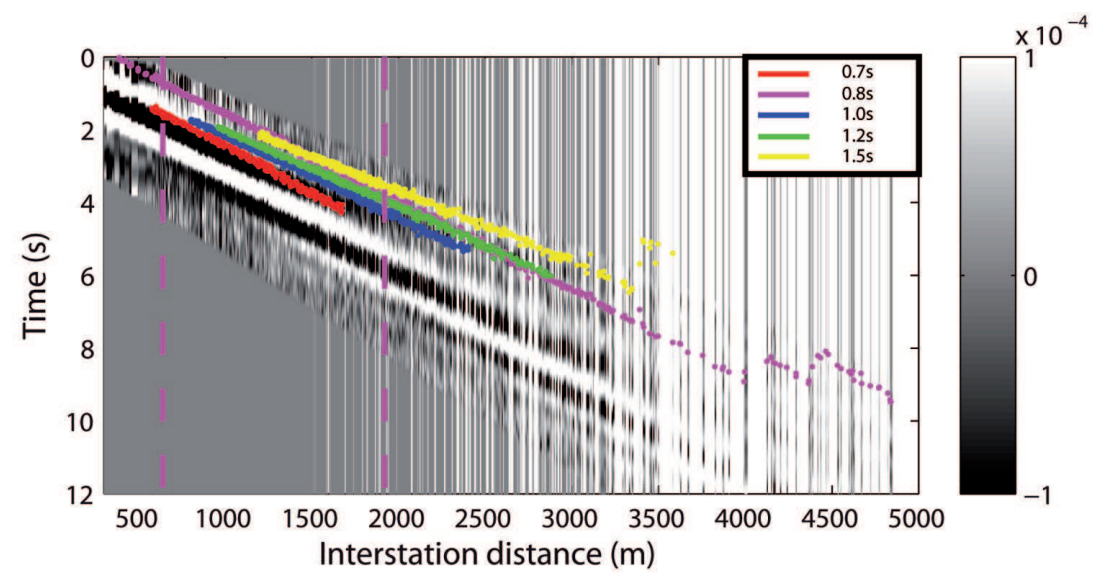

Figure 4 

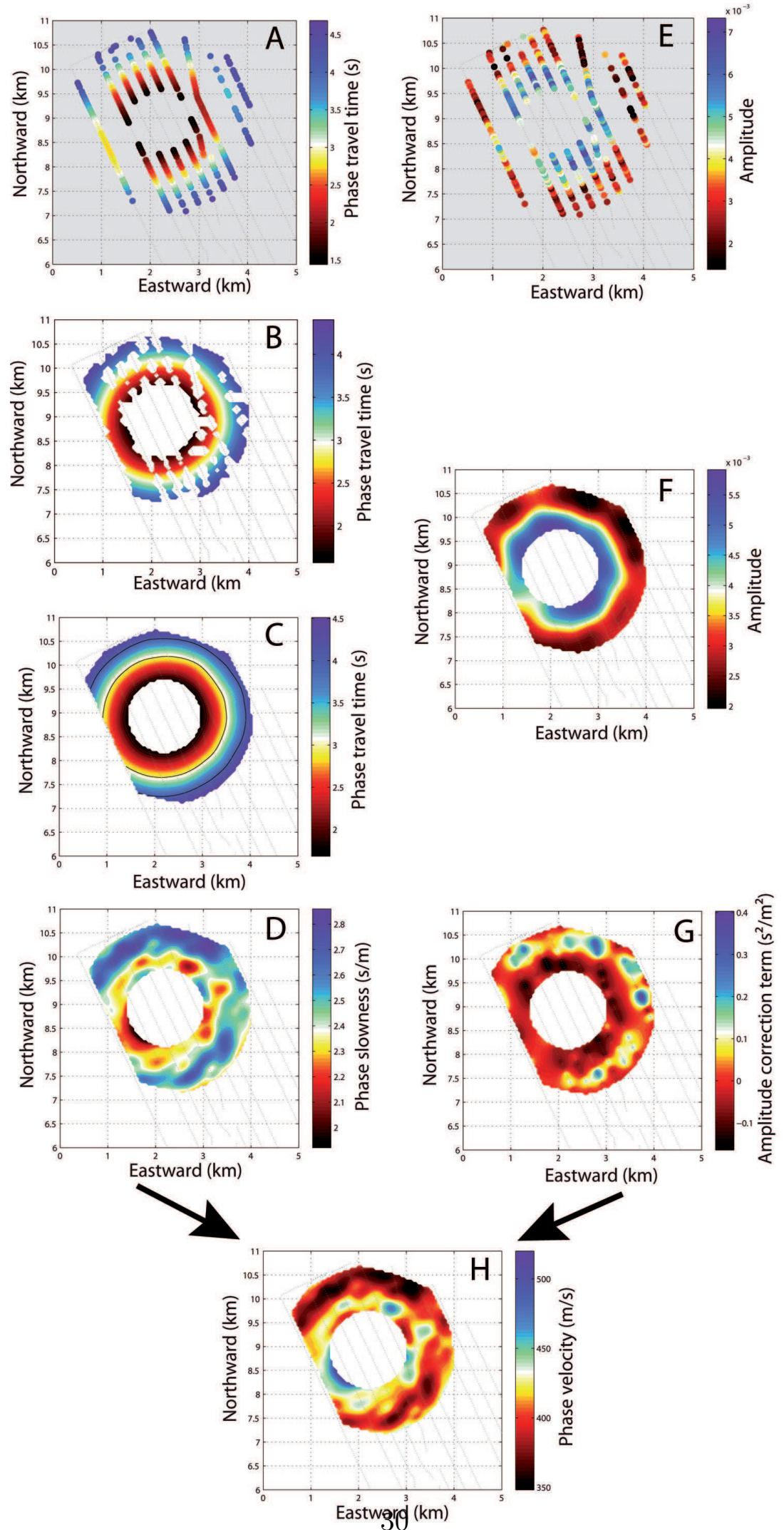

Figure 5 


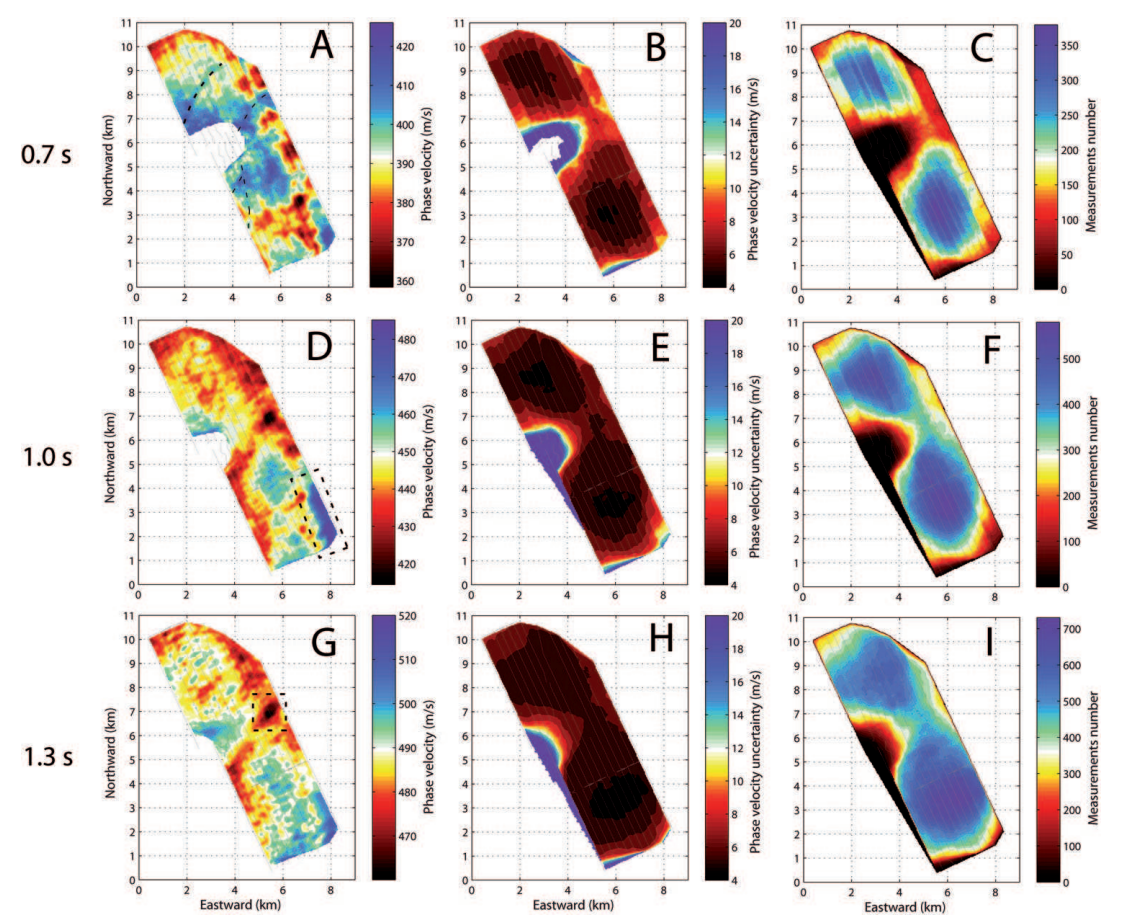

Figure 6 

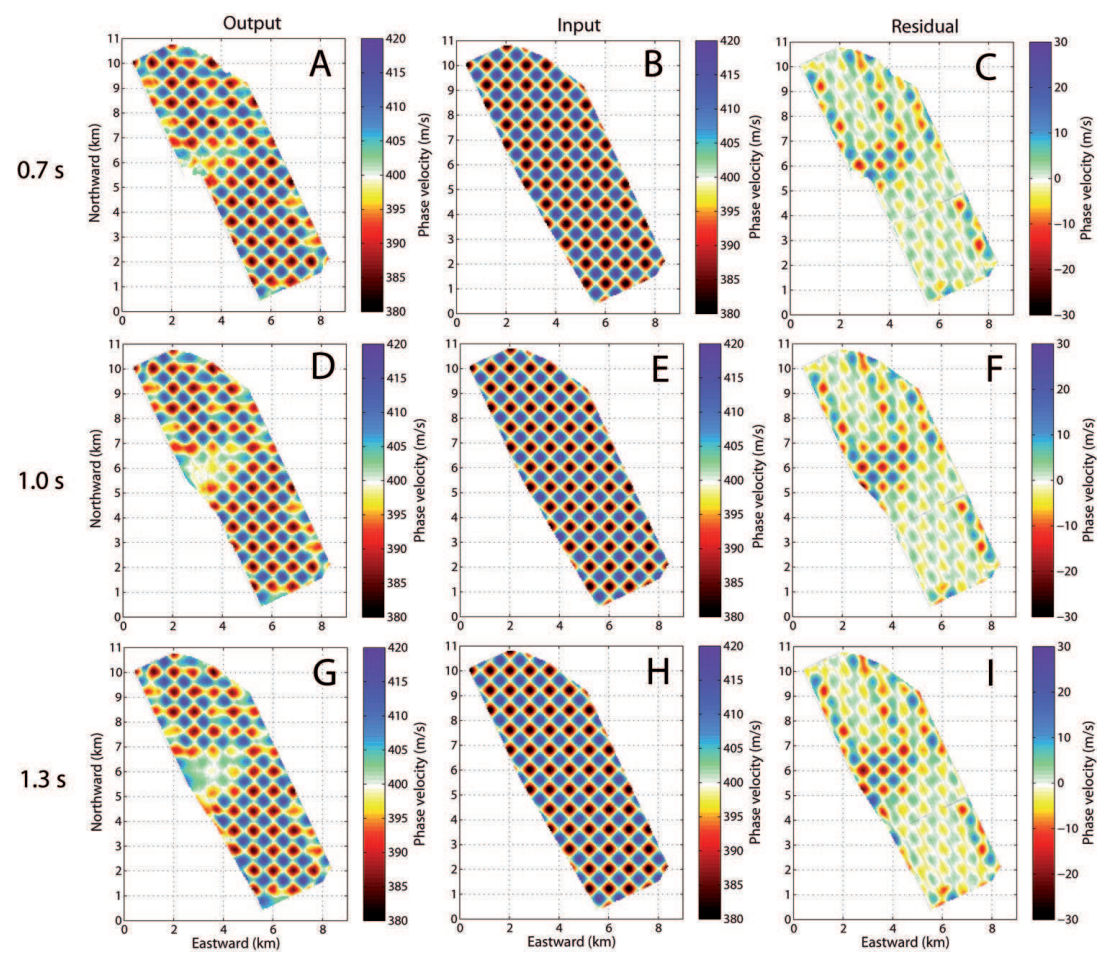

Figure 7

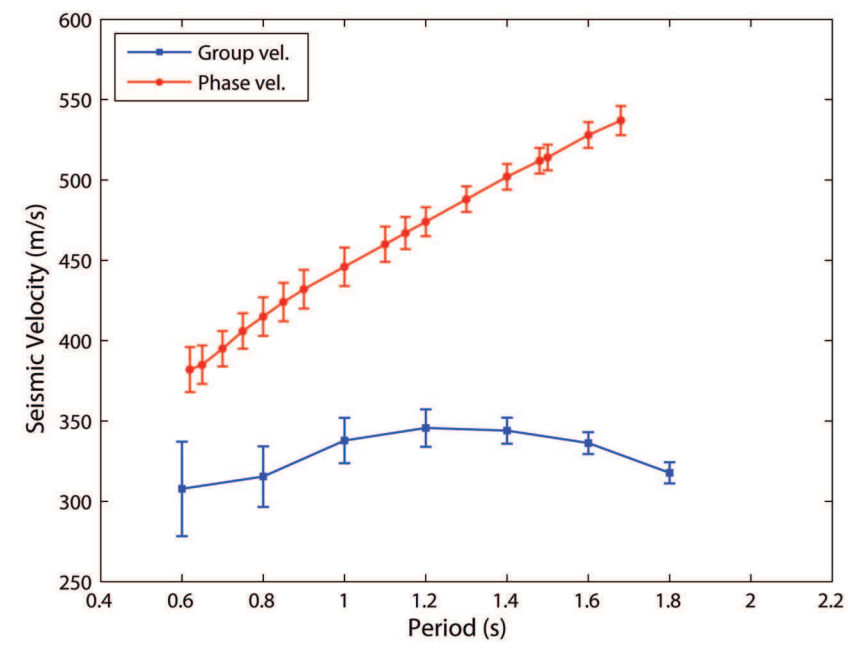

Figure 8 

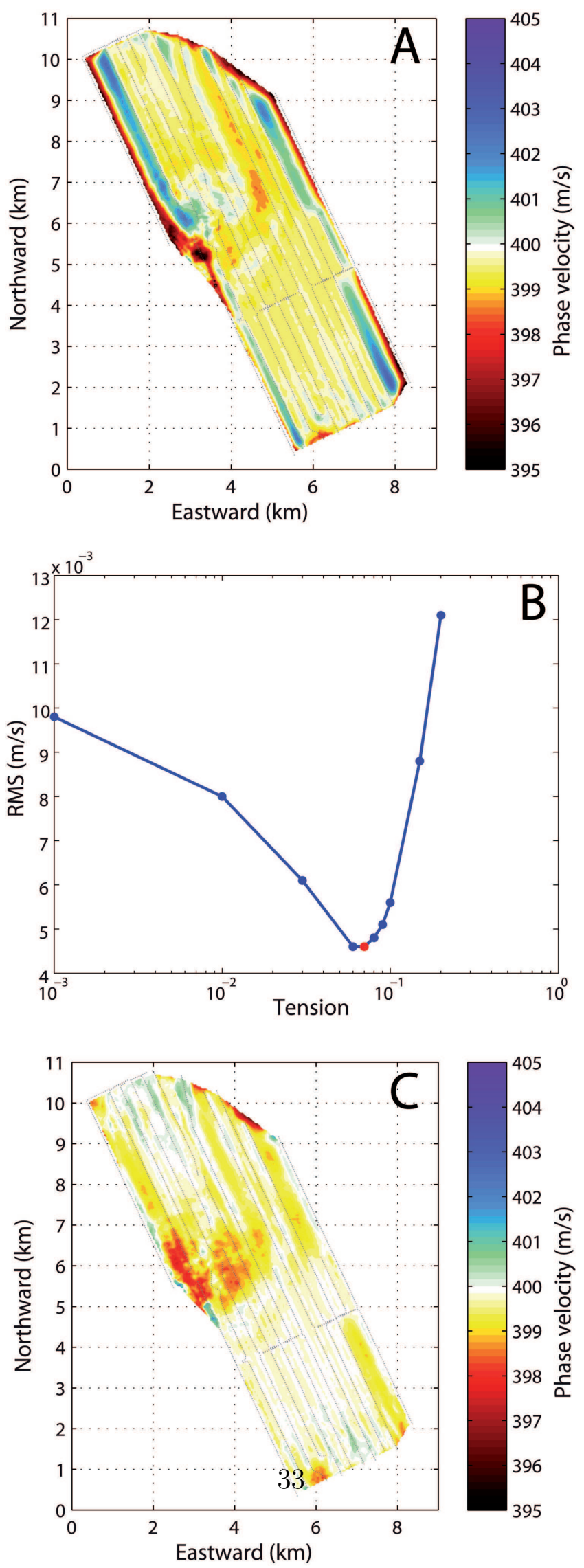

Figure 9 

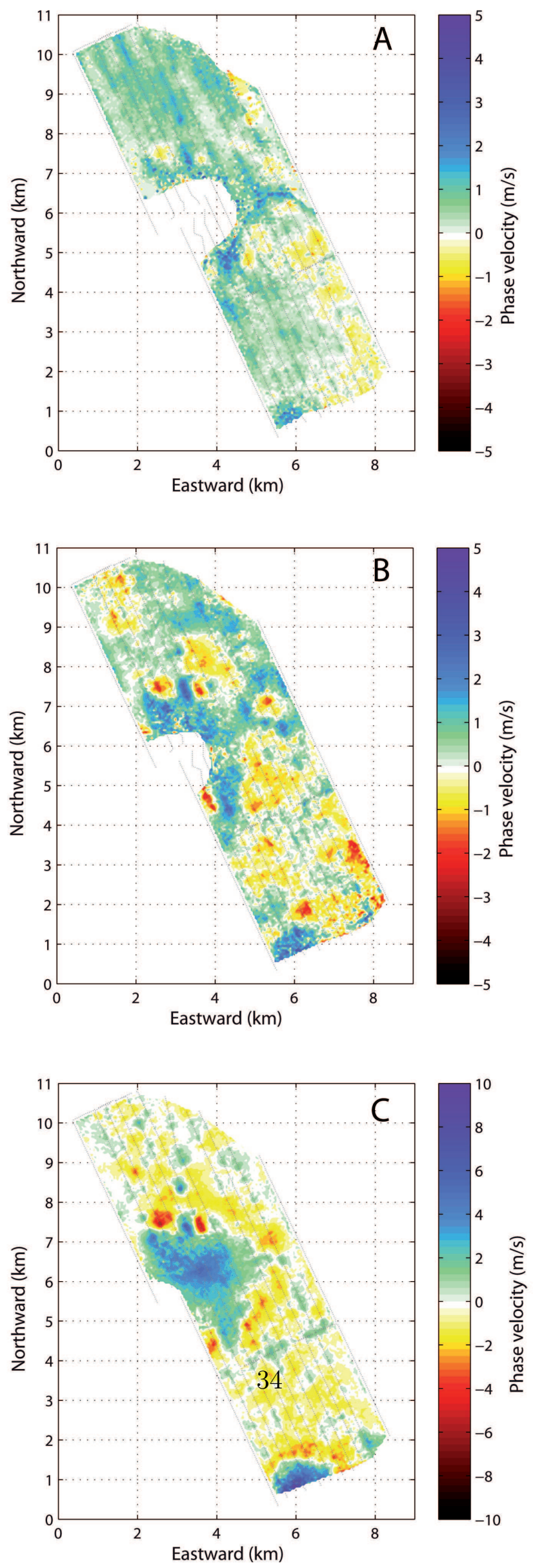